\title{
Ernährungs-Leitlinien für „Cancer Surviors“ - Wie gelingt Brustkrebsbetroffenen die Umsetzung?
}

\author{
Nutrition Guidelines for Cancer Survivors - How do Breast Cancer Survivors \\ Implement the Guidelines?
}

Autor

Institut
K. Kohlenberg-Müller

Hochschule Fulda - University of Applied Sciences
Schlüsselwörter

- Langzeit-Krebsüberlebende

- Brustkrebs

- Ernährungsstatus

- Lebensqualität

- Verzehrhäufigkeit

Keywords

- cancer survivorship

- breast cancer

- nutritional status

- quality of life

- food frequency
Bibliografie

DOI http://dx.doi.org/

10.1055/s-0035-1552756

Aktuel Ernahrungsmed 2015;

40: $335-340$

(c) Georg Thieme Verlag KG Stuttgart · New York

ISSN 0341-0501

Korrespondenzadresse:

Prof. Dr. Kathrin Kohlenberg-

Müller

Hochschule Fulda - University of

Applied Sciences

Marquardstraße 35

36039 Fulda

Tel.: +49(0)661/9640-378

kathrin.kohlenberg-

mueller@he.hs-fulda.de

\section{Zusammenfassung}

$\nabla$

Die Zahl der Krebsüberlebenden, insbesondere derjenigen in der rezidivfreien oder stabilen Lebensphase steigt und wurde in Deutschland in 2005/2006 auf 3,5 Mio. geschätzt [1]. Evidenzbasierte Empfehlungen der American Cancer Society (ACS) für die Ernährung und körperliche Aktivität von Krebsbetroffenen zielen auf die Reduktion des Krebsrisikos oder die Verbesserung des Outcomes von Krebsbetroffenen ab [2]. Brustkrebs ist die häufigste Krebserkrankung bei Frauen, über die Ernährung und körperliche Aktivität von Brustkrebsbetroffenen in Deutschland ist bisher wenig bekannt. In der vorliegenden Querschnittstudie wurden der Gesundheitsstatus und die Lebensqualität, der Ernährungsstatus, die körperliche Aktivität und das Verzehrverhalten von Brustkrebsbetroffenen in Selbsthilfegruppen, gynäkologischen Praxen sowie von Privatpersonen in verschiedenen Bundesländern mit einem standardisierten Fragebogen ermittelt. 236 Fragebögen wurden ausgewertet.

Der Zeitpunkt der Erstdiagnose liegt bei 75\% der Befragten mindestens 4 Jahre zurück. 59\% der Befragten sind älter als 60 Jahre. Bei 78\% der Befragten trat kein Lokalrezidiv auf. Der Gesundheitszustand und die körperliche Verfassung seit Behandlungsende werden nach Selbsteinschätzung mehrheitlich mit gut, die Lebensqualität sogar mit sehr gut bewertet. Einschränkungen bei körperlichen Tätigkeiten im Alltag werden von 58,5\% der Befragten angegeben. In der Altersklasse 6069 Jahre sind 34,0\% der Brustkrebsbetroffenen normalgewichtig, 49\% übergewichtig, 16,3\% adipös. Insgesamt sind von den Brustkrebsbetroffenen 35,6\% normalgewichtig, 45,3\% übergewichtig und $17,8 \%$ adipös. Bei $49,6 \%$ der Befragten erfolgten Gewichtszunahmen nach Abschluss der Therapie von $8,6 \pm 6,1 \mathrm{~kg}$. 44,9\% der Befragten geben ihre sportliche Aktivität mit 30 Minuten bis 2 Stunden pro Woche an. Obst und Gemüse werden

\section{Abstract \\ $\nabla$}

The number of cancer survivors, especially those that are recovered, including survivors who are disease free or who have stable disease is growing steadily and is estimated at 3.5 million in Germany in 2005/2006 [1]. Evidence based nutrition and physical activity guidelines for cancer survivors from the American Cancer Society (ACS) target on cancer risk reduction or on improving outcomes in cancer survivors [2]. Breast cancer is the most frequently diagnosed cancer in women, although little information about nutrition and physical activity of breast cancer survivors in Germany is available. The present cross-sectional study analyses the health status and quality of life, the nutritional status, physical activity and food frequency of breast cancer survivors by surveying self-help groups, gynaecological practices and private persons in different federal states. 236 standardized questionnaires were evaluated. $75 \%$ of participants were diagnosed more than 4 years prior to the study. $59 \%$ of participants were more than 60 years old. $78 \%$ of participants had not experienced a relapse/local recurrence. State of health and physical condition after the completion of treatment were mostly rated as good, quality of life was mostly rated even as very good. $58.5 \%$ of respondents stated to having had difficulties being physically active in daily life. Among the women aged $60-69$ years, $34.0 \%$ were of normal weight, $49.0 \%$ were overweight and $16.3 \%$ obese. Among all participants $35.6 \%$ were of normal weight, $45.3 \%$ were overweight and $17.8 \%$ obese. $49.6 \%$ of women had experienced a mean weight gain after the end of treatment of $8.6 \pm$ $6.1 \mathrm{~kg}$. $44.9 \%$ of participants reported weekly sporting activities of $0.5-2$ hours. Fruit and vegetables were consumed daily or nearly daily, whole grain products several times per week. $72.5 \%$ of participants had not been offered nutritional advice. 
im Mittel täglich bzw. fast täglich verzehrt, Vollkornprodukte mehrmals pro Woche. 72,5\% erhielten keine Angebote zur Ernährungsberatung.

Brustkrebsbetroffene in der rezidivfreien oder stabilen Lebensphase erreichen die für sie formulierten Empfehlungen zur Ernährung und körperlicher Aktivität mehrheitlich nicht. Es besteht ein hoher Bedarf an Interventionen zum Lebensstil.
Nutrition guidelines for cancer survivors have not been met by the majority of breast cancer survivors who are disease free or who have stable disease. There is a high demand for life style interventions.

\section{Einleitung}

$\nabla$

Krebs zählt zu der Gruppe der nicht übertragbaren Erkrankungen und ist für 20\% aller Todesfälle in der Europäischen Region der WHO verantwortlich. Mit mehr als 3 Mio. Neuerkrankungen und 1,7 Mio. Todesfällen jährlich ist Krebs nach den kardiovaskulären Erkrankungen die bedeutendste Ursache für Todesfälle und Morbidität in Europa [3]. In Deutschland wird die Zahl der Krebsbetroffenen, bei denen die Diagnose 5 bis 10 Jahre zurückliegt, auf 2,1 Mio., d.h. 2,6\% der Bevölkerung geschätzt, aus der 5-Jahres-Prävalenz ergeben sich weitere 1,4 Mio. Krebserkrankte (1,7\% der Bevölkerung) [1]. Im Vergleich dazu lebten in den USA in 2007 11,7 Mio. Menschen, d. h. 4\% der Bevölkerung mit Krebs [4]. 7 Mio. davon waren 65 Jahre und älter, 6,3 Mio., d. h. mehr als die Hälfte, waren Frauen. Bei 4,7 Mio. lag die Diagnose 10 Jahre oder länger zurück.

Von den Krebserkrankungen ist für Frauen das Mammakarzinom mit 70000 Neuerkrankungen pro Jahr in Deutschland die häufigste. Eine von 8 Frauen erkrankt daran im Laufe ihres Lebens. Jede 4. Frau ist bei der Diagnosestellung jünger als 55 Jahre. Die Überlebensraten sind seit den 1980er-Jahren kontinuierlich gestiegen, die Überlebenszeiten deutlich angestiegen. Für 2007/ 2008 wird die relative 5-Jahres-Überlebensrate für Frauen mit Mammakarzinom mit 87\% ausgewiesen [5].

Krebs wird heute als eine chronische Erkrankung eingestuft [6]. Als Krebsüberlebende (Cancer Survivors) gelten alle Personen, bei denen Krebs diagnostiziert wurde, d. h. vom Zeitpunkt der Diagnose bis zu ihrem Lebensende [4]. Dabei werden 3 verschiedene Phasen unterschieden, die aktive Behandlung und Regeneration (Phase 1), das Leben nach der Regeneration in der erkrankungsfreien oder stabilen Lebensphase (Phase 2) sowie der fortgeschrittene Krebs und das Lebensende (Phase 3) [2].

Brustkrebsbetroffene haben aber im Vergleich zu Nichtbetroffenen ein deutlich erhöhtes Risiko für Komorbiditäten durch bestimmte nicht übertragbare Erkrankungen. So wurde in einer retrospektiven Kohortenstudie für die brustkrebsspezifische Mortalität ein voll adjustiertes relatives Risiko (RR) von 1,24 für kardiovaskuläre Erkrankungen (95\%-KI 1,13-1,26), ein RR von 1,13 für einen weiteren Krebsvorfall (95\%-KI 1,13-1,26), ebenfalls ein RR von 1,13 für chronisch obstruktive Lungenerkrankungen $(95 \%-K I$ 1,05-1,22) und ein RR von 1,10 für Diabetes mellitus (95\%-KI 1,03-1,16) bestimmt. Die Autoren fordern daher, dass der Prävention von kardiovaskulären Erkrankungen in der Nachsorge von Brustkrebsbetroffenen Priorität beigemessen werden sollte [7].

Bereits 2003 wurden von der American Cancer Society (ACS) speziell für die Gruppe der Cancer Survivors Richtwerte für die Ernährung und körperliche Aktivität während und nach der Krebsbehandlung ausgesprochen. Evidenzbasierte Richtlinien für die Ernährung und körperliche Aktivität von Krebsbetroffenen wurden 2012 formuliert ( $\bullet$ Tab. 1). Diese zielen auf die Reduktion des Krebsrisikos oder die Verbesserung des Outcomes von Krebsbetroffenen ab [2, 8]. Gleichzeitig hat sich der World Cancer Re-
Tab. 1 Evidenzbasierte Richtlinien der American Cancer Society (2012) für die Ernährung und körperliche Aktivität von Krebsbetroffenen [2].

Es wird empfohlen, ein gesundes Körpergewicht anzustreben und aufrechtzuerhalten.

- Bei Übergewicht und Adipositas sollte der Konsum von hochkalorischen Lebensmitteln und Getränken begrenzt und die körperliche Aktivität erhöht werden, um den Gewichtsverlust zu fördern.

Es wird empfohlen, regelmäßig körperlich aktiv zu sein.

- Inaktivität sollte vermieden werden und nach der Diagnose sollte so schnell wie möglich zu einer normalen täglichen Aktivität zurückgekommen werden.

- Es sollte angestrebt werden, sich wenigstens 150 Minuten pro Woche zu bewegen.

- Krafttrainingübungen sollten wenigstens an 2 Tagen pro Woche durchgeführt werden.

Ein Ernährungsmuster reich an Gemüse, Obst und Vollkorn wird empfohlen.

- Es sollten die Empfehlungen der American Cancer Society zur Ernährung und körperlichen Aktivität für die Prävention von Krebs eingehalten werden.

Quelle [2]: Rock CL et al. CA: A Cancer Journal for Clinicians 2012; 62: 242-274

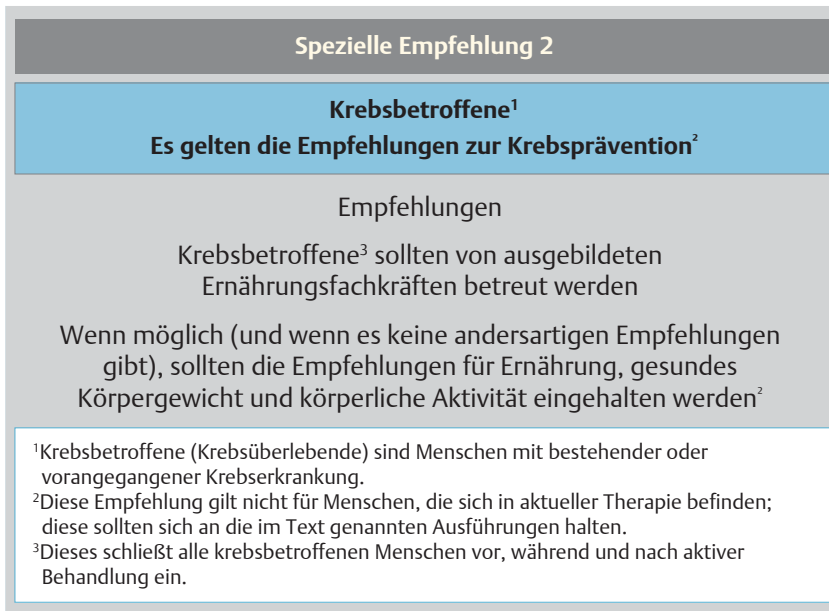

Abb. 1 World Cancer Research Fund/American Institute of Cancer Research - Second Expert Report (2007): Empfehlungen zur Krebsprävention [10].

search Fund (WCRF) und das American Institute for Cancer Research (AICR) in einem systematischen Review mit den Zusammenhängen zwischen Lebensmittel, Ernährung und Krebs auseinandergesetzt. In dem Update von 2007 werden neben den Empfehlungen zur Ernährung, körperlichen Aktivität und Krebsprävention auch spezielle Empfehlungen für Krebsbetroffene ausge-

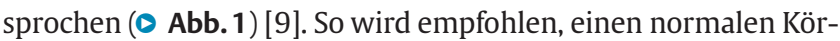
pergewichtsbereich anzustreben, den Verzehr energiedichter Lebensmittel zu begrenzen und überwiegend pflanzliche Lebensmittel zu verzehren. Begrenzt werden sollte der Verzehr von rotem Fleisch, der Konsum alkoholischer Getränke und der 


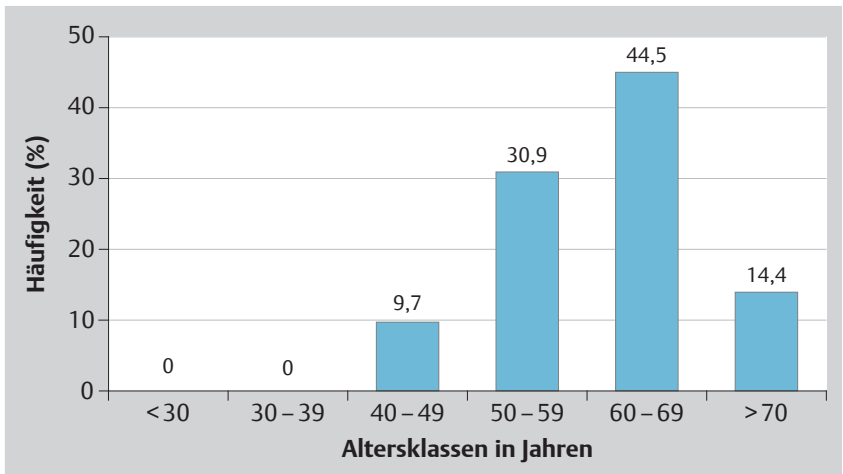

Abb.2 Altersverteilung der Brustkrebsbetroffenen $(n=236)([11]$, mit freundlicher Genehmigung des Umschau Zeitschriftenverlages, Wiesbaden).

Salzkonsum. Der Nährstoffbedarf sollte ausschließlich durch Lebensmittel gedeckt werden. Die Kurzfassung liegt in deutscher Sprache vor und kann über die Homepage der DGE abgerufen werden [10].

Die Auseinandersetzung mit Krebsbetroffenen gewinnt zunehmend an Bedeutung und Forschungsfragen zu Langzeit-Krebsüberlebenden (Cancer Survivorship Research) haben sich als ein neuer Forschungsbereich entwickelt. Über die Ernährung und körperliche Aktivität von Brustkrebsbetroffenen in Deutschland ist bisher jedoch wenig bekannt. Mit der vorliegenden Studie wurde hierzu eine erste Problemanalyse vorgenommen. Für Brustkrebsbetroffene in der rezidivfreien stabilen Lebensphase kann überprüft werden, wie Gesundheitsstatus und Lebensqualität, Ernährungsstatus, körperliche Aktivität und Verzehrverhalten in Deutschland aussehen.

\section{Methodik}

Für die Querschnittstudie wurde ein standardisierter Fragebogen mit Selbstangaben (Paper-Pencil-Verfahren) für Brustkrebsbetroffene zu den Themenkomplexen soziodemografische Daten, subjektive Einschätzung des Gesundheitszustands und der Lebensqualität nach der Krebserkrankung, Ernährungsstatus, Ernährungskenntnisse und Verzehrhäufigkeitsfragen sowie Angebot zur Ernährungsberatung mit überwiegend geschlossenen Fragen entwickelt. Die Befragung erfolgte überregional in Selbsthilfegruppen, gynäkologischen Praxen sowie bei Privatpersonen in den Bundesländern Baden-Württemberg, Bayern, Hessen und
Sachsen von Mai bis November 2012. 769 Fragebögen wurden persönlich, per E-Mail und Post an die Probandinnen abgegeben. Aus dem Rücklauf von 251 Fragebögen konnten 236 ausgewertet werden. Hierzu wurden die Evaluations-Software EvaSys und die Statistik-Software SPSS angewendet, offene Fragen wurden per Hand ausgewertet. Das detaillierte Konzept und Design dieser Studie wurde an anderer Stelle ausführlich beschrieben [11].

\section{Ergebnisse}

Von den 236 befragten Brustkrebsbetroffenen sind 59\% älter als 60 Jahre. Die Altersverteilung ist in 1 Abb. 2 wiedergegeben. Der Zeitpunkt der Erstdiagnose liegt bei 75\% der Befragten mindestens 4 Jahre zurück, bei $61 \%$ mehr als 6 Jahre. Im Rahmen der Therapie der Brustkrebserkrankung erfolgten bei $97 \%$ eine oder mehrere Operationen, 72\% erhielten eine Strahlentherapie, 62\% eine Chemotherapie. Bei 78\% der Befragten trat kein Lokalrezidiv auf.

Brustkrebsbetroffene schätzen ihren Gesundheitszustand und ihre körperliche Verfassung seit Behandlungsende mit gut ein. Die Lebensqualität seit Behandlungsende wird nach Selbsteinschätzung sogar mit sehr gut bewertet ( $\bullet$ Abb.3). Dabei geben $58,5 \%$ der Befragten an, dass sie sich bei körperlichen Tätigkeiten im Alltag eingeschränkt fühlen.

Die Verteilung der BMI-Kategorien in den verschiedenen Altersklassen zum Zeitpunkt der Befragung ist in $\bullet$ Abb. 4 dargestellt. Insgesamt sind von den Brustkrebsbetroffenen 35,6\% normalgewichtig, 45,3\% übergewichtig und 17,8\% adipös. 49,6\% der Befragten gaben Gewichtszunahmen von $8,6 \pm 6,1 \mathrm{~kg}$ nach Therapie-

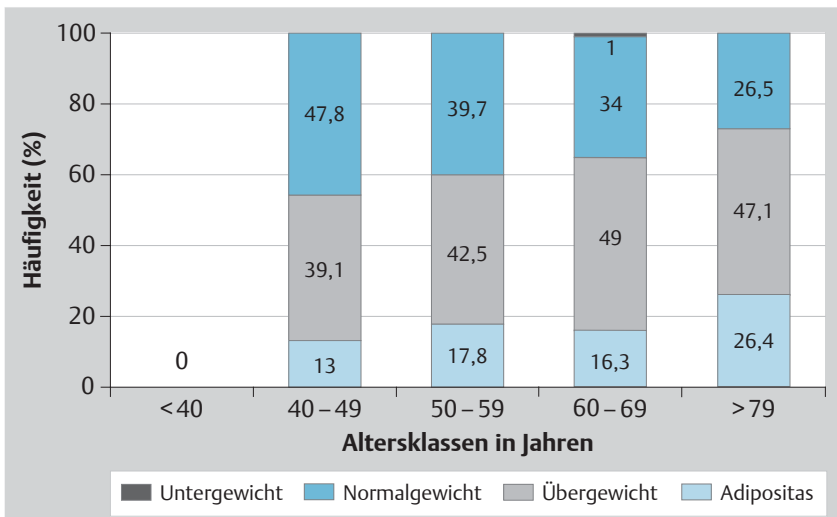

Abb.4 BMI-Verteilung in den Altersklassen ([11], mit freundlicher Genehmigung des Umschau Zeitschriftenverlages, Wiesbaden).

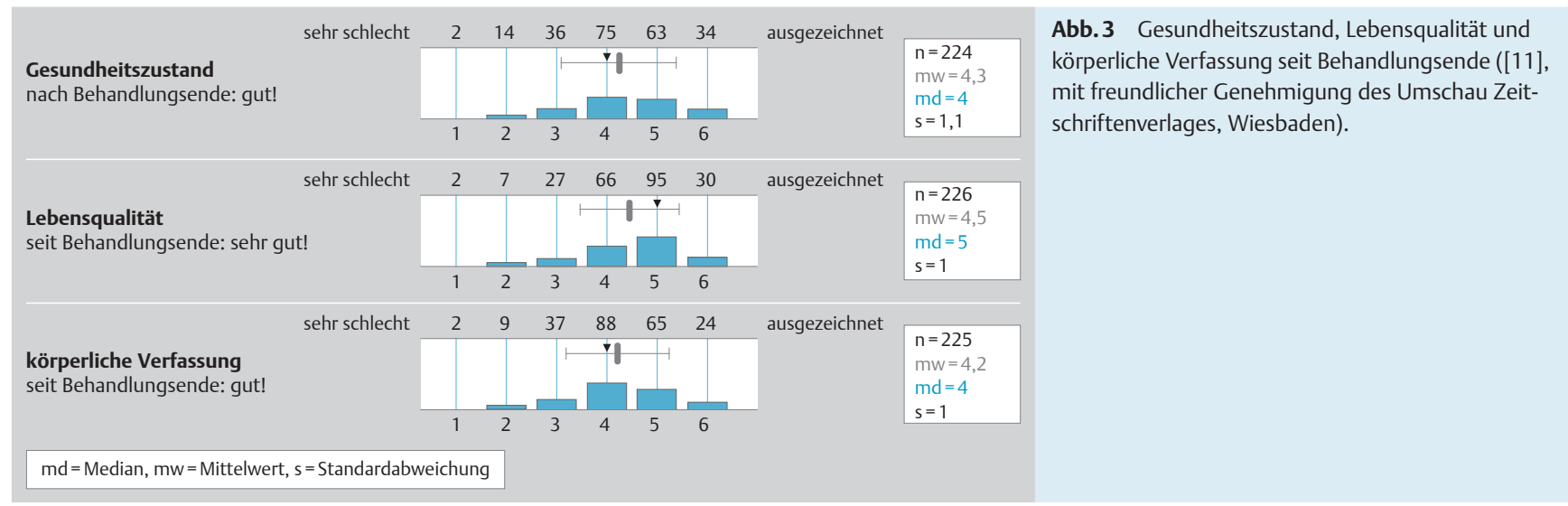




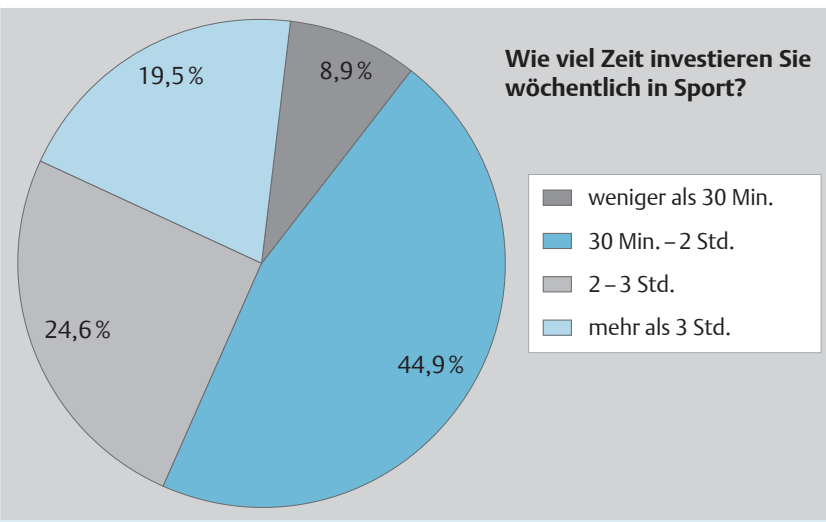

Abb.5 Sportliche Aktivität pro Woche ([11], mit freundlicher Genehmigung des Umschau Zeitschriftenverlages, Wiesbaden).

ende an. $69 \%$ von ihnen empfinden die Gewichtszunahme als ein Problem. Bei 14,8\% der Befragten kam es nach Abschluss der Therapie zur Gewichtsabnahme von $8,5 \pm 4,8 \mathrm{~kg}$. Diese wird von $97 \%$ nicht als ein Problem empfunden.

Die sportliche Aktivität wird von 44,9\% der Befragten mit 30 Minuten bis 2 Stunden pro Woche angegeben ( $\bullet$ Abb.5). Den Lebensmittelverzehr vor der Brustkrebserkrankung zeigt $\bullet$ Abb.6. Bei $57 \%$ der Befragten hat die Ernährung vor der Brustkrebserkrankung einen hohen Stellenwert. 56,5\% der Befragten reagieren nach eigenen Angaben auf die Brustkrebserkrankung mit einer Änderung der Ernährungsweise, vor allem diejenigen, die vorher nicht bewusst auf ihre Ernährung geachtet haben. So gaben $63 \%$ der Befragten an, sich seit Abschluss der Behandlung weniger fettreich zu ernähren. Auf die offene Frage, wie sich ihre Ernährungsweise seit Behandlungsende geändert hat, antworteten 121 Befragte, dass sie mehr Gemüse (49,6\%) und Obst (43,8\%) sowie weniger Fleisch und Wurst (33\%) verzehren.

Nur 27,1\% wurden von einem Arzt oder einem Ernährungsberater zur Ernährung befragt und erhielten während oder nach den Behandlungen ein Angebot zur Ernährungsberatung. 23,7\%, d.h. 87,5\% von denen, die ein Angebot zur Ernährungsbetreuung erhalten haben, nahmen dieses auch wahr.

\section{Diskussion}

$\nabla$

Die vorliegende Querschnittstudie liefert aussagekräftige Informationen zum Ernährungs- und Gesundheitsstatus sowie zur Ernährung und sportlichen Aktivität von Brustkrebsbetroffenen in der rezidivfreien stabilen Lebensphase. Von den Befragten leben drei Viertel schon mindestens 4 Jahre mit der chronischen Erkrankung. Die Altersverteilung und die angewendeten Therapieformen sind typisch für Brustkrebsbetroffene [12, 13], jedoch ist die Gruppe der über 70-Jährigen in der vorliegenden Arbeit etwas unterrepräsentiert. Die Spannbreite für das Risiko für Lokalrezidive bei Brustkrebs wird mit 4,2-12\% angegeben [14]. Unter den Befragten sind mit 19,5\% jedoch überproportional viele mit einem oder mehreren Rezidiven vertreten, sodass ein Selektionsbias vermutet werden kann.

Die Einschätzungen von Gesundheitszustand und Lebensqualität aus der subjektiven Sicht der Betroffenen gelten als wichtige Indikatoren für die Gesundheit. Treten eine oder mehrere chronische Erkrankungen auf, so verschlechtert sich die Lebensqualität signifikant [15]. Aufgrund von Restriktionen im Studiendesign können die vorliegenden Ergebnisse allerdings nicht mit dem über den SF-36V2 gewonnenen Referenzdaten zur gesundheitsbezogenen Lebensqualität von Erwachsenen in Deutschland [15] verglichen werden. Bemerkenswert ist jedoch, dass in der vorliegenden Studie die Brustkrebsbetroffenen ihre Lebensqualität seit Behandlungsende besser einschätzen als ihren Gesundheitszustand und ihre körperliche Verfassung. Bei Krebsbetroffenen werden positive Lebensveränderungen beschrieben, die als eine Auswirkung der Krebserkrankung einzustufen sind [16]. Gezeigt wurde für Krebsbetroffene auch ein sog. posttraumatisches Wachstum, d.h. Krebsbetroffene bewerten ihre Lebensqualität besser als Gesunde. Wichtig dabei ist es, zu berücksichtigen, dass sich teilweise der Maßstab verändert, mit dem die eigene Lebensqualität bewertet wird [17].

Der Ernährungsstatus, gemessen am BMI, ist bei zwei Drittel der befragten Brustkrebsbetroffenen durch Übergewicht und Adipositas charakterisiert, während Untergewicht nur eine sehr geringe Bedeutung hat. Damit fällt der Ernährungsstatus verglichen mit der Normalbevölkerung sogar etwas günstiger aus. So liegt bei Frauen in der Altersgruppe 60-69 Jahre in der für Deutschland repräsentativen DEGS1-Studie der Anteil der Normalgewichtigen um 5\% niedriger. Der Anteil der Adipösen beträgt bei den Brustkrebsbetroffenen nur 16,3\% im Vergleich zu 34,8\% in der Allgemeinbevölkerung [18]. Eine niedrigere Prävalenz von Adipositas bei Brustkrebsbetroffenen im Vergleich zur Normalbevölkerung wurde auch in einer anderen Studie [19] gezeigt.

Von etwa der Hälfte der Befragten werden markante mittlere Gewichtszunahmen nach Abschluss der Therapie im Umfang von $8,6 \pm 6,1 \mathrm{~kg}$ angegeben. Auch in der Life after Cancer Epidemiology (LACE) Study wurden bei $51 \%$ der 50- bis unter 60-jährigen Frauen Gewichtszunahmen nach der Diagnose Brustkrebs beschrie-

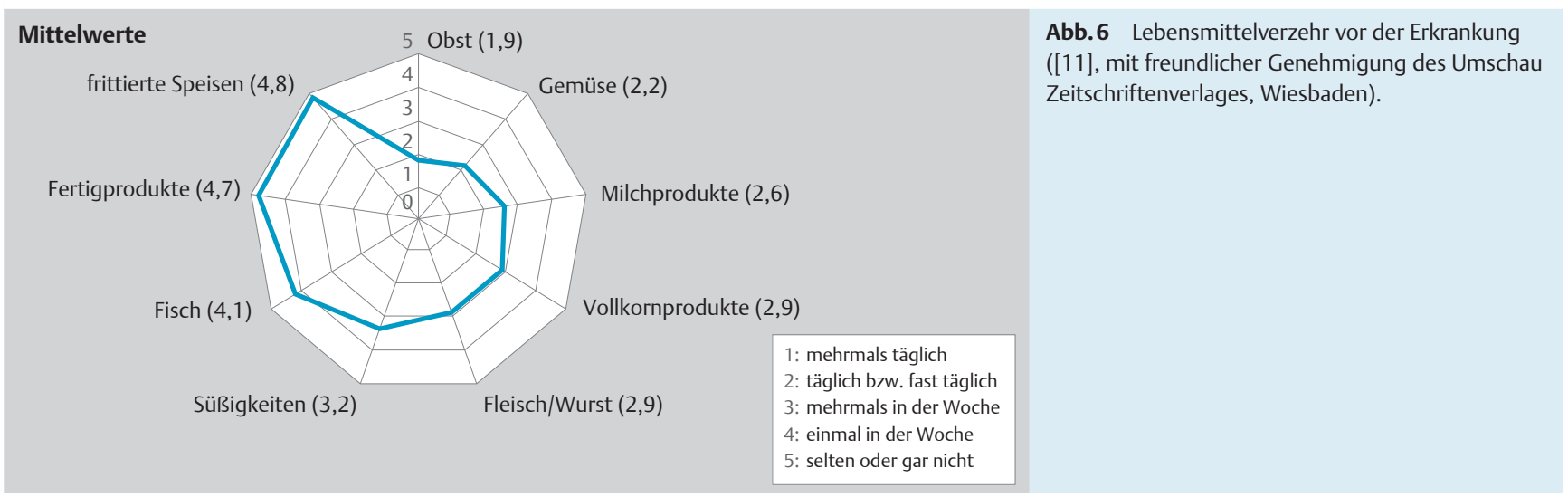


Tab.2 Zusammenfassung und Vergleich mit den evidenzbasierten Richtlinien der American Cancer Society (2012) für die Ernährung und körperliche Aktivität von Krebsbetroffenen [2].

Es wird empfohlen, ein gesundes Körpergewicht anzustreben und auf-
rechtzuerhalten.
- 65,1\% aller Studienteilnehmerinnen sind übergewichtig oder adipös.
- 49,6\% zeigen Gewichtszunahmen von 8,6 $\pm 6,1 \mathrm{~kg}$ nach Therapieende.
Es wird empfohlen, regelmäßig körperlich aktiv zu sein. Es sollte ange-
strebt werden, sich wenigstens 150 Minuten pro Woche zu bewegen.
- $24,6 \%$ betrieben 2 - 3 Stunden Sport pro Woche, $19,5 \%$ mehr als 3
Stunden Sport pro Woche.
- $53,8 \%$ betrieben weniger als 2 Stunden Sport pro Woche.
Ein Ernährungsmuster reich an Gemüse, Obst und Vollkorn wird emp-
fohlen
- Obst und Gemüse werden im Schnitt nicht mehrmals täglich sondern
nur täglich bzw. fast täglich verzehrt, Vollkornprodukte lediglich
mehrmals pro Woche.

Quelle [2]: Rock CL et al. CA: A Cancer Journal for Clinicians 2012; 62: 242-274

ben. Hier waren die Gewichtszunahmen gerade bei den jüngeren Brustkrebsbetroffenen besonders hoch [20].

Der Anteil sportlich Aktiver unter den Brustkrebsbetroffenen fällt im Vergleich zu der Normalbevölkerung höher aus, 44,1 \% der Befragten investieren 2-3 Stunden pro Woche oder mehr in Sport. In der DEGS1-Studie wurde in der Altersgruppe der 60- bis 69jährigen Frauen der Anteil derjenigen, die regelmäßig mindestens 2 Stunden pro Woche sportlich aktiv sind, mit $23,8 \%$ bestimmt. 42,4\% geben eine sportliche Aktivität von bis zu 2 Stunden pro Woche an [21]. Dabei kann eine Überschätzung des Aktivitätsverhaltens der Befragten nicht ausgeschlossen werden.

Die Brustkrebsbetroffenen geben an, im Mittel täglich bzw. fast täglich Obst und Gemüse zu verzehren. Inwieweit dieses Ernährungsmuster von der Normalbevölkerung abweicht, kann nicht beurteilt werden. 60- bis 69-jährige Frauen haben nach der DEGS1-Studie von allen Frauen den höchsten Obst- und Gemüsekonsum. Dies wird als ein Hinweis für ein steigendes Gesundheitsbewusstsein im Alter interpretiert. Sie konsumieren im Mittel 3,7 Portionen Obst, Gemüse und Säfte pro Tag. Die empfohlenen 5 Portionen pro Tag erreichen jedoch nur 18,6\% der Frauen in der Altersgruppe 60-69 Jahre [22].

Insgesamt zeigen sich bei den befragten Brustkrebsbetroffenen deutliche Unterschiede zum Ernährungsstatus und Gesundheitsverhalten der Allgemeinbevölkerung. Adipositasprävalenz, körperliche Aktivität und das Verzehrverhalten fallen bei den Brustkrebsbetroffenen günstiger aus. Da Krebsbetroffene durch ihre chronische Erkrankung nachweislich höhere Komorbiditätsraten aufweisen und zudem Ernährung und körperliche Aktivität für die Gesundheitsförderung von hoher Bedeutung sind, wird ein hoher Bedarf an Lebensstilinterventionen, die diese vulnerable Population auch erreicht, formuliert [8].

Zwischen den vorliegenden Daten und den Empfehlungen für die Ernährung und körperliche Aktivität, die für Krebsbetroffene ausgesprochen werden, besteht jedoch eine große Diskrepanz: Es wird empfohlen, ein gesundes Körpergewicht anzustreben und aufrechtzuerhalten, rund 63\% der befragten Brustkrebsbetroffenen sind übergewichtig oder adipös, ca. 50\% haben an Gewicht zugenommen. Die Empfehlung, regelmäßig körperlich aktiv zu sein, wird von 54\% der Befragten nicht erreicht. Das empfohlene Ernährungsmuster reich an Gemüse, Obst und Vollkorn wird angestrebt, jedoch im Mittel nicht realisiert ( Tab.2). Die im World Cancer Research Fund/American Institute of Cancer Research-Report formulierte Empfehlung, Krebsbetroffene vor, während und nach aktiver Behandlung sollten von ausgebildeten Ernährungsfachkräften betreut werden, wird weitestgehend nicht umgesetzt. 72,5\% der Befragten gaben an, kein Angebot zur Ernährungsberatung erhalten zu haben.

\section{Fazit}

\section{$\nabla$}

Brustkrebsbetroffene in der rezidivfreien stabilen Lebensphase erreichen die für sie formulierten Empfehlungen zur Ernährung und körperlichen Aktivität mehrheitlich nicht. Es besteht ein hoher Interventionsbedarf für diese große und ansteigende Gruppe gerade auch vor dem Hintergrund, die Krankheitslast nicht übertragbarer Erkrankungen zu reduzieren.

\section{Danksagung}

Die Autorin dankt den Absolventinnen des Studiengangs Oecotrophologie (B.Sc.) Nadine Bader, Melanie Billinger, Anna Meister, Katrin Pfeiffer, Julia Schneider und Sonja Voigt für die Durchführung und Auswertung dieser Studie sowie den zahlreichen Frauen, die an dieser Erhebung teilgenommen haben.

\section{Interessenkonflikt}

\section{$\nabla$}

Die Autorin gibt an, dass kein Interessenkonflikt besteht.

\section{Literatur}

1 Schilling G, Arnold D. Therapiefolgen bei Krebs-Langzeitüberlebenden. Bundesgesundheitsbl 2012; 55: 493-500

2 Rock CL, Doyle C, Demark-Wahnefried $W$ et al. Nutrition and physical activity guidelines for cancer survivors. CA: A Cancer Journal for Clinicians 2012; 62: $242-274$

3 WHO Regional Office for Europe. Cancer. http://www.euro.who.int/en/ health-topics/noncommunicable-diseases/cancer/cancer (accessed 13 May 2015)

4 Centers for Disease Control and Prevention. Cancer survivors - United States, 2007. MMWR Morb Mortal Wkly Rep 2011; 60: 269-272

5 Zentrum für Krebsregisterdaten. Krebs - Brustkrebs. http://www. krebsdaten.de/Krebs/DE/Content/Krebsarten/Brustkrebs/brustkrebs_ node.html;jsessionid=186017426293163662E600D4BC932B58.2 cid284 (accessed 4 Jun 2013)

6 Scheidt-Nave C. Chronische Erkrankungen - Epidemiologische Entwicklung und die Bedeutung für die Öffentliche Gesundheit. Public Health Forum 2010; 18: 2.e1

7 PatnaikJL, Byers T, DiGuiseppi C et al. Cardiovascular disease competes with breast cancer as the leading cause of death for older females diagnosed with breast cancer: a retrospective cohort study. Breast Cancer Res 2011; 13: R64

8 Robien K, Demark-Wahnefried W, Rock CL. Evidence-Based Nutrition Guidelines for Cancer Survivors: Current Guidelines, Knowledge Gaps, and Future Research Directions. Journal of the American Dietetic Association $2011 ; 111: 368-375$

9 World Cancer Research Fund/American Institute for Cancer Research. Food, Nutrition, Physical Activity, and the Prevention of Cancer: a Global Perspective. Washington: 2007

10 World Cancer Research Fund/American Institute for Cancer Research. Food, Nutrition, Physical Activity, and the Prevention of cancer: a Global perspective. Zusammenfassung. http://www.dge.de/uploads/media/WCRF-Report-summary-de.pdf (accessed 8 Jan 2015)

11 Bader N, Billinger M, Pfeiffer K et al. Leben mit Brustkrebs - Ernährung in der rezidiv-freien stabilen Lebensphase. Ernahrungs Umschau 2014; 61: $42-49$

12 Robert Koch-Institut, ed. Verbreitung von Krebserkrankungen in Deutschland. Entwicklung der Prävalenzen zwischen 1990 und 2010. Beiträge zur Gesundheitsberichterstattung des Bundes

13 Giersiepen K, Heitmann C, Janhsen K, Lange C. Brustkrebs. Aus der Reihe "Gesundheitsberichterstattung des Bundes" - Themenheft 25, Mai 2005. Internet: https://www.gbe-bund.de/gbe10/ergebnisse.prc_tab? 
fid=9508\&suchstring=\&query_id=\&sprache=D\&fund_typ=TXT\&meth ode $=\& \mathrm{vt}=\&$ verwandte $=1$ \&page_ret $=0 \&$ seite $=1 \& \mathrm{p} \_l \mathrm{fd} \_n \mathrm{r}=4 \& \mathrm{p} \_$news $=$ \&p_sprachkz=D\&p_uid=gast\&p_aid=8266646\&hlp_nr=2\&p_janein=J (accessed 9 Jan 2015)

14 Valdivieso M. Cancer Survivors in the United States: A Review of the Literature and a Call to Action. Int J Med Sci 2012; 9: 163-173

15 Ellert $U$, Kurth $B$. Gesundheitsbezogene Lebensqualität bei Erwachsenen in Deutschland. Bundesgesundheitsbl 2013; 56: 643-649

16 Zebrack BJ, Yi J, Petersen $L$ et al. The impact of cancer and quality of life for long-term survivors. Psycho-Oncology 2008; 17: 891-900

17 Weis J, Faller H. Psychosoziale Folgen bei Langzeitüberlebenden einer Krebserkrankung. Bundesgesundheitsbl 2012; 55: 501 -508
18 Mensink G, Schienkiewitz A, Scheidt-Nave C. Übergewicht und Adipositas in Deutschland: Werden wir immer dicker? DEGS-Symposium. Robert Koch-Institut; 2012: 6-9

19 Herman DR, Ganz PA, Petersen L et al. Obesity and cardiovascular risk factors in younger breast cancer survivors: The Cancer and Menopause Study (CAMS). Breast Cancer Res Treat 2005; 93: 13-23

20 Caan B, Sternfeld B, Gunderson E et al. Life After Cancer Epidemiology (LACE) Study: a cohort of early stage breast cancer survivors (United States). Cancer Causes Control 2005; 16: 545-556

21 Krug S, Jordan S, Mensink G et al. Körperliche Aktivität. Bundesgesundheitsbl 2013; 56: 765-771

22 Mensink G, Truthmann J, Rabenberg M et al. Obst- und Gemüsekonsum in Deutschland. Bundesgesundheitsbl 2013; 56: 779-785 